\title{
Outcomes of long-term nivolumab and subsequent chemotherapy in Japanese patients with head and neck cancer: 2-year follow-up from a multicenter real-world study
}

\author{
Ryuji Yasumatsu ${ }^{1} \cdot$ Yasushi Shimizu $^{2} \cdot$ Nobuhiro Hanai $^{3} \cdot$ Shin Kariya ${ }^{4} \cdot$ Tomoya Yokota $^{5} \cdot$ Takashi Fujii $^{6}$. \\ Kiyoaki Tsukahara ${ }^{7} \cdot$ Mizuo Ando $^{8,27} \cdot$ Kenji Hanyu $^{9} \cdot$ Tsutomu Ueda $^{10} \cdot$ Hitoshi Hirakawa $^{11}$. Shunji Takahashi ${ }^{12}$. \\ Takeharu Ono ${ }^{13}$. Daisuke Sano ${ }^{14} \cdot$ Moriyasu Yamauchi ${ }^{15} \cdot$ Akihito Watanabe $^{16} \cdot$ Koichi Omori $^{17}$. \\ Tomoko Yamazaki $^{18} \cdot$ Nobuya Monden $^{19} \cdot$ Naomi Kudo $^{20} \cdot$ Makoto Arai $^{21} \cdot$ Syuji Yonekura ${ }^{22} \cdot$ Takahiro Asakage $^{23}$. \\ Takahiro Nekado $^{24} \cdot$ Takayuki Yamada $^{25} \cdot$ Akihiro Homma $^{26}$
}

Received: 13 April 2021 / Accepted: 30 September 2021 / Published online: 13 November 2021

(c) The Author(s) 2021

\begin{abstract}
Background We have previously reported the effectiveness and safety of nivolumab in patients with head and neck cancer (HNC) in real-world clinical practice in Japan. Here, we report long-term outcomes from this study in the overall population and subgroups stratified by subsequent chemotherapy.

Methods In this multicenter, retrospective observational study, Japanese patients with recurrent or metastatic (R/M) HNC receiving nivolumab were followed up for 2 years. Effectiveness endpoints included overall survival (OS), OS rate, progression-free survival (PFS), and PFS rate. Safety endpoints included the incidence of immune-related adverse events (irAEs). Results Overall, 256 patients received a median of 6.0 doses (range: 1-52) of nivolumab over a median duration of 72.5 days (range: 1-736). Median OS was 9.5 months [95\% confidence interval (CI) 8.2-12.0] and median PFS was 2.1 months (95\% CI 1.8-2.7). A significant difference between 2-year survivors $(n=62)$ and non-2-year survivors was observed by median age $(P=0.0227)$ and ECOG PS $(P=0.0001)$. Of 95 patients who received subsequent chemotherapy, $54.7 \%$ received paclitaxel \pm cetuximab. The median OS and PFS from the start of paclitaxel \pm cetuximab were 6.9 months (95\% CI 5.9-11.9) and 3.5 months (95\% CI 2.3-5.5), respectively. IrAEs were reported in $17.2 \%$ of patients. Endocrine (7.0\%) and lung (4.3\%) disorders were the most common irAEs; kidney disorder $(n=1)$ was newly identified in this follow-up analysis.

Conclusions Results demonstrated the long-term effectiveness of nivolumab and potential effectiveness of subsequent chemotherapy in patients with R/M HNC in the real-world setting. Safety was consistent with that over the 1-year follow-up.
\end{abstract}

Keywords Nivolumab $\cdot$ Long-term survivors $\cdot$ Recurrent or metastatic head and neck cancer $\cdot$ Subsequent chemotherapy

\section{Introduction}

Nivolumab is a fully human immunoglobulin G4 monoclonal antibody that targets programmed cell death protein-1. Nivolumab was approved in March 2017 in Japan for the treatment of unresectable recurrent or distant metastatic $(\mathrm{R} / \mathrm{M})$ head and neck cancer (HNC) that had progressed following chemotherapy. This approval was based on the survival benefits and manageable safety profile demonstrated

Akihiro Homma

ak-homma@med.hokudai.ac.jp

Extended author information available on the last page of the article in the global phase 3 CheckMate 141 study in patients with R/M squamous cell carcinoma of the head and neck (SCCHN) whose disease had progressed within 6 months after platinum-based chemotherapy [1]. The 2-year followup results of the CheckMate 141 study confirmed the longterm survival benefits of nivolumab [2]. In the CheckMate 141 study, long-term (2-year) follow-up data were reported for the overall population [2], as well as for the Asian subpopulation [3]. However, only 27 Japanese patients were enrolled in the study. Therefore, the long-term survival data of nivolumab in Japanese patients need to be investigated in a real-world setting.

In the 2-year follow-up analysis of CheckMate 141, the baseline characteristics between long-term survivors (LTSs), 
who were alive (in survival follow-up) at 2 years, and the overall population was investigated, but no difference was observed [2]. On the other hand, there are no reports related to the baseline characteristics between LTSs and non-LTSs in Japanese clinical practice. Therefore, prognostic factors for long-term survival in Japanese patients remain unknown. In Japan, nivolumab use is not limited to patients with squamous cell carcinoma (SCC); it is also used in patients with non-SCC, who were not included in CheckMate 141 [4]. The subgroup analysis results for SCC and non-SCC at the 1-year follow-up have been reported previously, whereby the effectiveness between patients with SCC and non-SCC was similar, with no statistically significant differences [5]. Considering the approved indication of nivolumab in Japan, effectiveness and prognostic factors according to SCC and non-SCC in long-term survivors need to be evaluated.

Recently, several studies have demonstrated the efficacy of subsequent chemotherapy in HNC following immunotherapy [6-9]. Saleh et al. reported an objective response rate (ORR) of $30 \%$ in patients who received chemotherapy after disease progression on immunotherapy, which was three to five times higher than that in those who had received chemotherapy as a second-line treatment in clinical trials [6]. Similar studies in Japan have demonstrated the efficacy of subsequent chemotherapy following immunotherapy in patients with HNC; however, these studies included only a small number of Japanese patients [7-9]. Furthermore, in patients with R/M HNC in whom disease progresses following first-line therapy, treatment options remain heterogeneous, and treatment patterns and outcomes show substantial variations across countries [10]. Therefore, the outcomes of subsequent chemotherapy following nivolumab treatment in the Japanese clinical setting is an important clinical question to be answered.

Although immune-related adverse events (irAEs) are frequently reported within a few weeks to months of initiating treatment with immune checkpoint inhibitors, delayedonset irAEs sometimes occur after treatment discontinuation [11]. The onset of irAEs after nivolumab discontinuation has not been elucidated in patients with $\mathrm{R} / \mathrm{M} \mathrm{HNC}$ in a real-world clinical setting. Thus, effectiveness and safety data of nivolumab treatment over a long-term follow-up are warranted.

We recently reported the 1-year results from a real-world study in Japanese patients with HNC receiving nivolumab treatment to fill the data gap between clinical trials and realworld settings [5, 12]. Here, we report the long-term (2-year) effectiveness and safety results from this real-world study, including the effectiveness of subsequent chemotherapy.

\section{Patients and methods}

\section{Study design}

This was a multicenter, non-interventional, retrospective study conducted at 23 centers across Japan [5]. This study was conducted in accordance with the Ministerial Ordinance (number 171, issued on December 20, 2004) on Good Post-marketing Study Practice, Ministry of Health, Labour and Welfare, Japan, and the ethical principles of the Declaration of Helsinki. The study protocol was reviewed and approved by the institutional review board/ independent ethics committee at each study site. Informed consent was not obtained; however, patients were given the opportunity to decline to have their clinical records used for research (opt-out consent provision). This study was registered at ClinicalTrials.gov (NCT03569436) and in the University hospital Medical Information Network Clinical Trials Registry (UMIN-CTR; UMIN000032600).

Patients with R/M HNC with disease progression on or after platinum-based therapy and who were treated with nivolumab at least once for the first time between Jul 1, 2017, and Dec 31, 2017, were included in the study, except those who had participated in a clinical trial assessing antineoplastic therapy [5]. Patient data from baseline until the most recent visit were collected in electronic case report forms from medical charts. The data cutoff date was 2 years after the first treatment with nivolumab in each patient.

\section{Assessments and endpoints}

Primary endpoints were effectiveness and safety [5]. Effectiveness endpoints included best overall response (BOR), progression-free survival (PFS), and overall survival (OS) according to the investigator-assessed Response Evaluation Criteria in Solid Tumours (RECIST) 1.1 criteria [13]. Subgroup analyses were conducted to investigate the differences in baseline characteristics between 2-year survivors and non-2-year survivors and the effectiveness of subsequent chemotherapy. At the 2-year follow-up, patients who were alive were defined as 2-year survivor, while those who were not alive were defined as non-2-year survivors.

Safety endpoint included the incidence of irAEs. AEs were collected if they occurred up to 100 days after the last dose of nivolumab or up to the survey date, whichever was earlier. AEs were classified according to the International Council for Harmonisation of Technical Requirements for Pharmaceuticals for Human Use and Medical Dictionary for Regulatory Activities Japanese edition (MedDRA/J) 
version 21.0. The onset of irAEs was considered delayed in case of onset beyond 4 weeks of nivolumab discontinuation in patients who received subsequent systemic therapy other than nivolumab.

\section{Statistical analysis}

Effectiveness and safety analyses were conducted in all patients who received $\geq 1$ dose of nivolumab. Continuous variables were summarized as number, mean, and standard deviation; and categorical variables, as number and percentage. OS and PFS were estimated using the Kaplan-Meier method and expressed as the number and proportion of patients who survived to a specific point in time and median duration, with the corresponding twosided $95 \%$ confidence interval (CI). 95\% CI was calculated using the LOGLOG transformation. For the subgroup analysis to investigate differences between 2-year survivors and non-2-year survivors by baseline characteristics, $P$ values were calculated using the Chi-square test and the Wilcoxon rank-sum test. Statistical analyses were conducted using SAS version 9.4 (SAS Institute, Tokyo, Japan).

\section{Results}

\section{Patient characteristics}

Overall, 256 patients from 23 clinical institutions were registered. Table 1 shows baseline demographics and patient characteristics. At the 2-year follow-up, $8.6 \%(n=22)$ of enrolled patients were still receiving nivolumab treatment.
Table 1 Baseline characteristics stratified by survival status

\begin{tabular}{|c|c|c|c|c|}
\hline \multirow[t]{2}{*}{ Characteristic } & \multirow{2}{*}{$\begin{array}{l}\text { All patients } \\
(n=256)\end{array}$} & \multirow[b]{2}{*}{$\begin{array}{l}\text { Non-2-year survivors } \\
(n=194)\end{array}$} & \multirow[b]{2}{*}{$\begin{array}{l}\text { 2-year survivors } \\
(n=62)\end{array}$} & \multirow[b]{2}{*}{$P$ value } \\
\hline & & & & \\
\hline Sex & & & & 0.7417 \\
\hline Male & 202 (78.9) & $154(79.4)$ & $48(77.4)$ & \\
\hline Female & $54(21.1)$ & $40(20.6)$ & $14(22.6)$ & \\
\hline Age*, years, median (range) & $66(20-84)$ & $65(20-84)$ & $67(33-79)$ & 0.0227 \\
\hline \multicolumn{5}{|l|}{ Age category } \\
\hline$<65$ years & $114(44.5)$ & $94(48.5)$ & $20(32.3)$ & \\
\hline$\geq 65$ and $<75$ years & $118(46.1)$ & $79(40.7)$ & $39(62.9)$ & \\
\hline$\geq 75$ years & $24(9.4)$ & $21(10.8)$ & $3(4.8)$ & \\
\hline ECOG PS* & & & & 0.0001 \\
\hline 0 & $118(46.1)$ & $78(40.2)$ & $40(64.5)$ & \\
\hline 1 & $97(37.9)$ & $77(39.7)$ & $20(32.3)$ & \\
\hline$\geq 2$ & $31(12.1)$ & $31(16.0)$ & $0(0.0)$ & \\
\hline Unknown & $10(3.9)$ & $8(4.1)$ & $2(3.2)$ & \\
\hline \multicolumn{5}{|l|}{ Primary site } \\
\hline Hypopharynx & $64(25.0)$ & $50(25.8)$ & $14(22.6)$ & 0.6133 \\
\hline Oral cavity & $56(21.9)$ & $44(22.7)$ & $12(19.4)$ & 0.5814 \\
\hline Oropharynx & $40(15.6)$ & $30(15.5)$ & $10(16.1)$ & 0.9001 \\
\hline Salivary gland & $23(9.0)$ & $17(8.8)$ & $6(9.7)$ & 0.8265 \\
\hline Larynx & $21(8.2)$ & $17(8.8)$ & $4(6.5)$ & 0.5637 \\
\hline Maxillary sinus & $14(5.5)$ & $11(5.7)$ & $3(4.8)$ & 0.8021 \\
\hline Nasopharynx & $19(7.4)$ & $11(5.7)$ & $8(12.9)$ & 0.0586 \\
\hline Others & $19(7.4)$ & $14(7.2)$ & $5(8.1)$ & 0.8245 \\
\hline \multicolumn{5}{|l|}{ Previous treatment } \\
\hline Surgery & $176(68.8)$ & $132(68.0)$ & $44(71.0)$ & 0.6652 \\
\hline Cetuximab & $155(60.5)$ & $124(63.9)$ & $31(50.0)$ & 0.0510 \\
\hline Chemoradiation therapy & $144(56.3)$ & $107(55.2)$ & $37(59.7)$ & 0.5320 \\
\hline Radiation therapy & 94 (36.7) & $74(38.1)$ & $20(32.3)$ & 0.4026 \\
\hline
\end{tabular}

*Significant differences were observed between non-2-year survivors and 2-year survivors

Data are $n(\%)$ unless specified otherwise

ECOG PS Eastern Cooperative Oncology Group performance status 
Of the 234 patients (91.4\%) who discontinued nivolumab treatment at 2 years, $179(76.5 \%)$ had disease progression.

\section{Treatment exposure}

Seventy patients $(27.3 \%)$ received nivolumab as the first-line treatment for R/M HNC, $110(43.0 \%)$ as second-line, and 76 (29.7\%) as third- or later-line treatment [5]. Patients received a median of 6.0 doses (range: 1-52) of nivolumab over a median duration of 72.5 days (range: $1-736$ ) of nivolumab treatment.

\section{Effectiveness of nivolumab in the overall population}

Table 2 shows the effectiveness of nivolumab represented by the BOR, ORR, and disease control rate (DCR). The ORR was $16.1 \%$ (95\% CI 11.6-21.6) and the DCR was $43.0 \%$ (95\% CI 36.5-49.8; Table 2). The median OS was 9.5 months (95\% CI 8.2-12.0) and the estimated 24-month OS rate was $30.1 \%$ (Fig. 1a). The median PFS was 2.1 months (95\% CI 1.8-2.7) and the estimated 24-month PFS rate was $8.9 \%$ (Fig. 1b).

\section{Effectiveness by subgroups}

Of the 256 patients, 62 were alive at the 2-year follow-up analysis. The BOR, ORR, and DCR in 60 patients of 2-year survivors are shown in Table 2 . The BOR in 2-year survivors was complete response in three (5.0\%), partial response in 25 (41.7\%), and stable disease in $21(35.0 \%)$ patients (Table 2). The ORR and DCR in 2-year survivors were $46.7 \%(95 \%$ CI 33.7-60.0) and 81.7\% (95\% CI 69.6-90.5), respectively (Table 2). Significant differences between 2-year survivors and non-2-year survivors were observed (Table 1 ) by age $(P=0.0227)$ and Eastern Cooperative Oncology Group performance status (ECOG PS; $P=0.0001$ ). There were no patients with ECOG PS $\geq 2$ in 2-year survivors, while $16 \%$ of patients had ECOG PS $\geq 2$ in non-2-year survivors.

This study included 29 patients with non-SCC in addition to patients with SCC $(n=217)$. Effectiveness according to histological type (SCC vs. non-SCC) is shown in Online Resources 1-3. Effectiveness was similar between both groups, with no statistically significant differences; for instance, the median OS was 9.1 months $(95 \% \mathrm{CI}$ 7.4-10.4) in patients with SCC and 15.1 months [95\% CI 4.5-not reached (NR)] in patients with non-SCC. A total of 50 patients with SCC were alive at the 2-year follow-up analysis. Significant differences between 2-year survivors and non-2-year survivors in SCC were observed by age $(P=0.0326)$, nasopharynx as primary site $(P=0.0388)$, and ECOG PS $(P=0.0007)$ (Online Resource 4).

\section{Effectiveness of subsequent chemotherapy}

Ninety-five patients (37.1\%) received chemotherapy following nivolumab treatment (Table 3). Of these, $54.7 \%$ $(n=52)$ received paclitaxel \pm cetuximab as subsequent chemotherapy (8/52 patients received only paclitaxel; data not shown). Figure 2 shows the median OS and PFS from the start date of subsequent chemotherapy. In patients who received subsequent chemotherapy, the median OS was 9.8 months (95\% CI 6.9-12.8; Fig. 2a) and the median PFS was 4.3 months (95\% CI 2.3-5.3; Fig. 2b). In patients who received paclitaxel \pm cetuximab as subsequent chemotherapy, the median OS was 6.9 months (95\% CI 5.9-11.9) and the estimated 24-month OS rate was $30.2 \%$ (Fig. 2a); the median PFS was 3.5 months (95\% CI 2.3-5.5) and the estimated 24-month PFS rate was $22.9 \%$ (Fig. 2b). In patients who received platinum-based therapy or S-1 therapy as subsequent chemotherapy, the median OS was 11.5 months (95\% CI 5.2-NR) and 20.3 months (95\% CI 1.9-20.3), respectively, with an estimated 24-month OS rate of $23.8 \%$ and $0 \%$, respectively (Fig. 2a); the median PFS was
Table 2 Effectiveness of nivolumab treatment during the 2-year follow-up period

\begin{tabular}{lll}
\hline & Overall $(n=256)$ & 2-year survivors $(n=62)$ \\
\hline $\begin{array}{l}\text { Evaluable patients } \\
\text { BOR }\end{array}$ & $223(87.1)$ & $60(23.4)$ \\
$\quad$ Complete response & $3(1.3)$ & $3(5.0)$ \\
Partial response & $33(14.8)$ & $25(41.7)$ \\
Stable disease & $60(26.9)$ & $21(35.0)$ \\
Progressive disease & $127(57.0)$ & $11(18.3)$ \\
ORR & $36(16.1) 95 \%$ CI 11.6-21.6 & $28(46.7) 95 \%$ CI 33.7-60.0 \\
DCR & $96(43.0) 95 \%$ CI 36.5-49.8 & $49(81.7) 95 \%$ CI 69.6-90.5 \\
\hline
\end{tabular}

Data are $n(\%)$

Response rates were calculated in evaluable patients

$B O R$ best overall response, $C I$ confidence interval, $D C R$ disease control rate, $O R R$ objective response rate 
Fig. 1 Kaplan-Meier curves in the overall population: a OS and b PFS. Survival curves were plotted based on the last survival confirmation date.

Two-year survivors are shown as censored at 24 months. $C I$ confidence interval, $O S$ overall survival, $P F S$ progression-free survival (a)

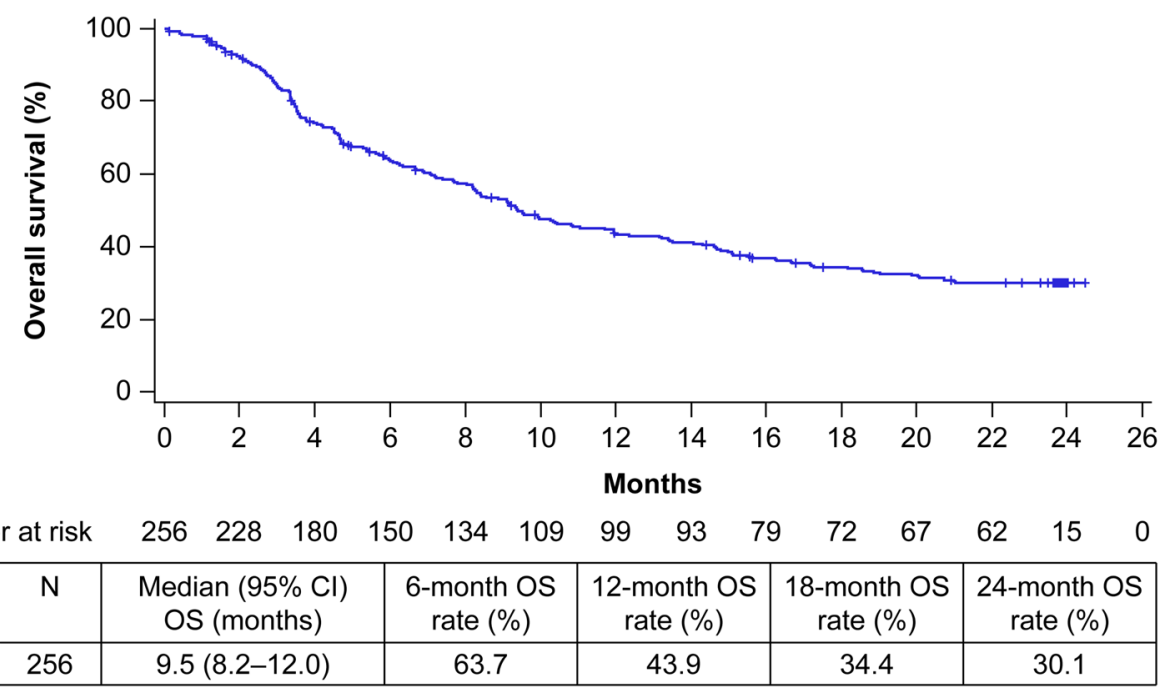

(b)

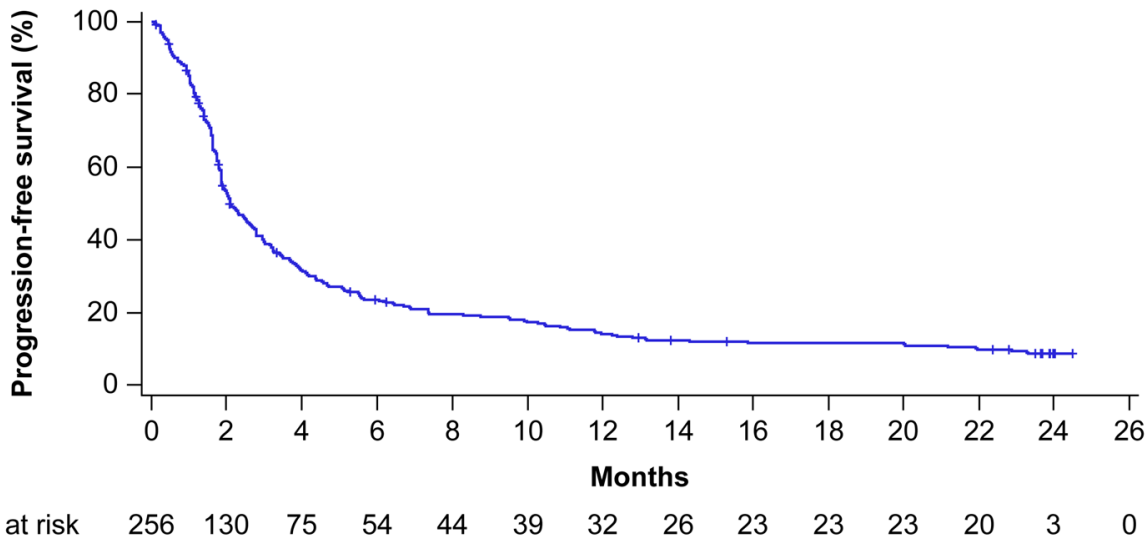

$\begin{array}{lllllllllllllll}\text { Number at risk } & 256 & 130 & 75 & 54 & 44 & 39 & 32 & 26 & 23 & 23 & 23 & 20 & 3 & 0\end{array}$

\begin{tabular}{|c|c|c|c|c|c|}
\hline $\mathrm{N}$ & $\begin{array}{c}\text { Median (95\% Cl) } \\
\text { PFS (months) }\end{array}$ & $\begin{array}{c}\text { 6-month PFS } \\
\text { rate (\%) }\end{array}$ & $\begin{array}{c}\text { 12-month PFS } \\
\text { rate (\%) }\end{array}$ & $\begin{array}{c}\text { 18-month PFS } \\
\text { rate (\%) }\end{array}$ & $\begin{array}{c}\text { 24-month PFS } \\
\text { rate (\%) }\end{array}$ \\
\hline 256 & $2.1(1.8-2.7)$ & 23.6 & 14.2 & 11.5 & 8.9 \\
\hline
\end{tabular}

Table 3 Effectiveness of subsequent chemotherapy

\begin{tabular}{lllll}
\hline & Overall & $\begin{array}{l}\text { Paclitaxel } \pm \\
\text { cetuximab }\end{array}$ & S-1 therapy & Platinum-based therapy \\
\hline $\begin{array}{l}\text { Total patients } \\
\text { Evaluable patients, }\end{array}$ & $95(100.0)$ & $52(54.7)$ & $13(13.7)$ & $12(12.6)$ \\
$n(\%)$ & $42(44.2)$ & $6(6.3)$ & $10(10.5)$ \\
BOR & & & $1(16.7)$ & $0(0.0)$ \\
Complete response & $4(5.7)$ & $3(7.1)$ & $1(16.7)$ & $4(40.0)$ \\
Partial response & $19(27.1)$ & $12(28.6)$ & $0(0.0)$ & $2(20.0)$ \\
Stable disease & $17(24.3)$ & $13(31.0)$ & $4(66.7)$ & $4(40.0)$ \\
Progressive disease & $30(42.9)$ & $14(33.3)$ & $2(33.3) 95 \%$ CI 4.3-77.7 & $4(40.0) 95 \%$ CI 12.2-73.8 \\
ORR & $23(32.9) 95 \%$ CI 22.1-45.1 & $15(35.7) 95 \%$ CI 21.6-52.0 & \\
DCR & $40(57.1) 95 \%$ CI 44.7-68.9 & $28(66.7) 95 \%$ CI 50.5-80.4 & $2(33.3) 95 \%$ CI 4.3-77.7 & $6(60.0) 95 \%$ CI 26.2-87.8 \\
\hline
\end{tabular}

Data are $n(\%)$

Response rates were calculated in evaluable patients

$B O R$ best overall response, $C I$ confidence interval, $D C R$ disease control rate, $O R R$ objective response rate 
Fig. 2 Kaplan-Meier curves in patients who received chemotherapy following nivolumab treatment: a OS and b PFS. $C I$ confidence interval, $N R$ not reached, $O S$ overall survival, $P F S$ progression-free survival (a)

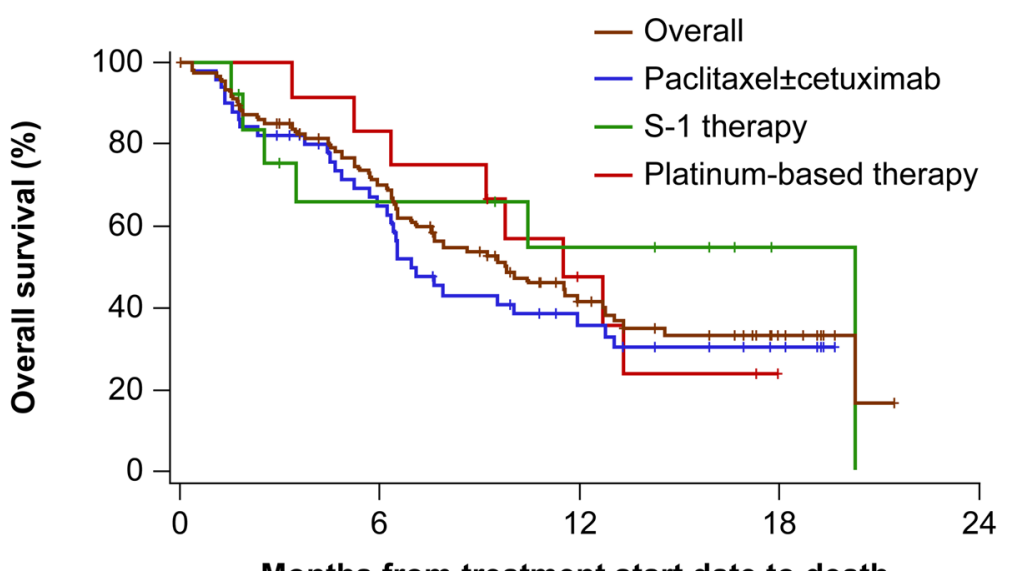

Number at risk

Overall

$\begin{array}{ll}\text { Paclitaxel } \pm \text { cetuximab } & 95 \\ & 52\end{array}$

S-1 therapy

13

Platinum-based therapy

\begin{tabular}{|l|c|c|c|c|c|c|}
\hline & $\mathrm{N}$ & $\begin{array}{c}\text { Median (95\% Cl) } \\
\text { OS (months) }\end{array}$ & $\begin{array}{c}\text { 6-month } \\
\text { OS rate (\%) }\end{array}$ & $\begin{array}{c}\text { 12-month } \\
\text { OS rate (\%) }\end{array}$ & $\begin{array}{c}\text { 18-month } \\
\text { OS rate (\%) }\end{array}$ & $\begin{array}{c}\text { 24-month } \\
\text { OS rate (\%) }\end{array}$ \\
\hline Overall & 95 & $9.8(7.1-12.8)$ & 70.2 & 41.6 & 33.4 & 16.7 \\
\hline Paclitaxeltcetuximab & 52 & $6.9(5.9-11.9)$ & 65.1 & 35.7 & 30.2 & 30.2 \\
\hline S-1 therapy & 13 & $20.3(1.9-20.3)$ & 66.1 & 55.1 & 55.1 & 0 \\
\hline Platinum-based therapy & 12 & $11.5(5.2-\mathrm{NR})$ & 83.3 & 47.6 & 23.8 & 23.8 \\
\hline
\end{tabular}

(b)

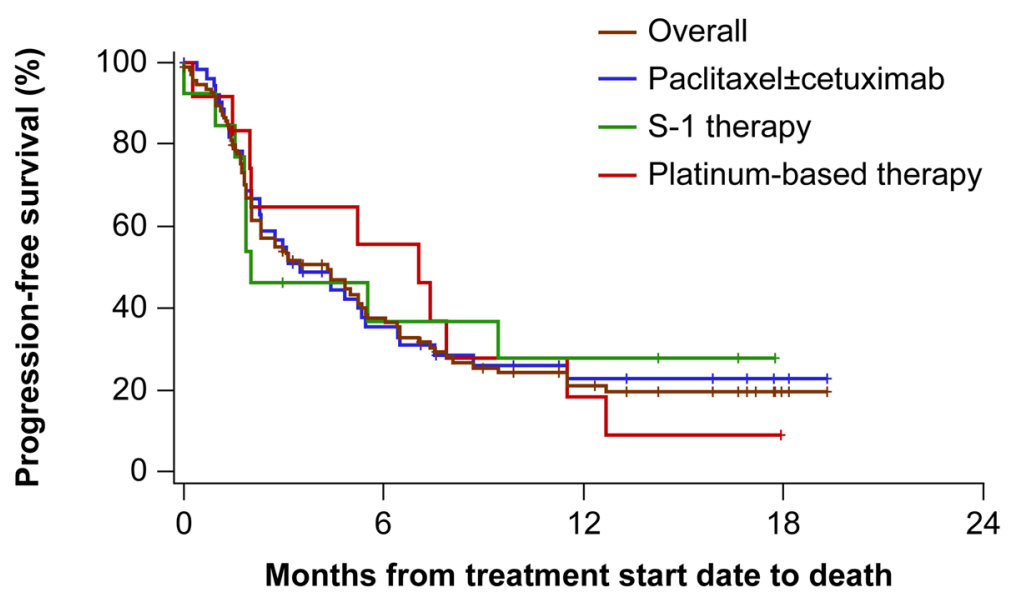

Number at risk

Overall 95

Paclitaxel \pm cetuximab $\quad 52$

S-1 therapy

52
13

Platinum-based therapy

12

$61 \quad 27$

$30 \quad 13$

$7 \quad 5$

90

-

10

$0 \quad 0$

\begin{tabular}{|l|c|c|c|c|c|c|}
\hline & $\mathrm{N}$ & $\begin{array}{c}\text { Median (95\% CI) } \\
\text { PFS (months) }\end{array}$ & $\begin{array}{c}\text { 6-month } \\
\text { PFS rate (\%) }\end{array}$ & $\begin{array}{c}\text { 12-month } \\
\text { PFS rate (\%) }\end{array}$ & $\begin{array}{c}\text { 18-month } \\
\text { PFS rate (\%) }\end{array}$ & $\begin{array}{c}\text { 24-month } \\
\text { PFS rate (\%) }\end{array}$ \\
\hline Overall & 95 & $4.3(2.3-5.3)$ & 37.6 & 21.1 & 19.5 & 19.5 \\
\hline Paclitaxelıcetuximab & 52 & $3.5(2.3-5.5)$ & 35.6 & 22.9 & 22.9 & 22.9 \\
\hline S-1 therapy & 13 & $2.0(1.5-N R)$ & 36.9 & 27.7 & 27.7 & 27.7 \\
\hline Platinum-based therapy & 12 & $7.0(1.4-11.5)$ & 55.6 & 18.5 & 9.3 & 9.3 \\
\hline
\end{tabular}

7.0 months (95\% CI 1.4-11.5) and 2.0 months (95\% CI 1.5-NR), respectively, with an estimated 24-month PFS rate of $9.3 \%$ and $27.7 \%$, respectively (Fig. 2b).

\section{Safety}

The overall incidence of any-grade irAEs was $17.2 \%(n=44$; Table 4). The most frequently reported irAEs were endocrine 
Table 4 Incidence of irAEs stratified by severity

\begin{tabular}{lcr}
\hline irAE category $(n=256)$ & All grades & Grade $\geq 3$ \\
\hline All patients with irAE & $44(17.2)$ & $18(7.0)$ \\
Lung disorder & $11(4.3)$ & $6(2.3)$ \\
Liver disorder & $7(2.7)$ & $3(1.2)$ \\
Skin disorder & $7(2.7)$ & $2(0.8)$ \\
Endocrine disorder & $18(7.0)$ & $4(1.6)$ \\
Neurological disorder & $0(0.0)$ & $0(0.0)$ \\
Kidney disorder & $1(0.4)$ & $0(0.0)$ \\
Gastrointestinal disorder & $4(1.6)$ & $2(0.8)$ \\
Blood disorder & $2(0.8)$ & $1(0.4)$ \\
Others & $6(2.3)$ & $2(0.8)$ \\
\hline
\end{tabular}

Data are $n(\%)$

irAE immune-related adverse event

disorders in 18 (7.0\%), lung disorders in 11 (4.3\%), and liver and skin disorders in seven $(2.7 \%)$ patients each. Kidney disorder $(n=1)$ was newly identified in this follow-up analysis. Grade $\geq 3$ irAEs were reported in $18(7.0 \%)$ patients (Table 4); lung disorders were the most common (2.3\%) grade $\geq 3$ irAE. Of 44 patients with irAEs of any grade, 39 reported irAEs during nivolumab treatment and five following nivolumab discontinuation (Fig. 3).

\section{Discussion}

To our knowledge, this study provides the first long-term, real-world results of nivolumab in a large population with $\mathrm{R} / \mathrm{M}$ HNC in Japan. The effectiveness of subsequent chemotherapies after nivolumab discontinuation as well as delayed irAEs were investigated to improve treatment management in patients with R/M HNC. In addition, patient characteristics that may impact long-term survival were also investigated.

The long-term real-world effectiveness of nivolumab in this study was comparable to that observed in the overall study population [2] and Asian subpopulation [3] in CheckMate 141 (2-year OS rate: 30.1, 16.9, 22.7\%; median OS duration: 9.5, 7.7, 12.1 months; and ORR: 16.1, 13.3, 26.1\%, respectively). The 2-year OS rate and ORR in the current study were higher than those observed in CheckMate 141. This difference could be due to the inclusion of patients with primary nasopharyngeal cancer [7.4\% $(n=19)]$ in the current study. Patients with the nasopharynx as the primary site generally demonstrated a better OS rate and ORR than those with $\mathrm{HNC}$ at other primary sites in a subgroup analysis of the 1-year follow-up results from this study [5], but this patient population was not included in CheckMate 141. Thus, the difference in OS rate and ORR for the overall population between the current study and CheckMate 141 could be attributed to the inclusion and exclusion of patients with primary nasopharyngeal cancer. However, the efficacy of nivolumab in nasopharyngeal cancer should be elucidated in other confirmatory clinical trials.

In CheckMate 141 and the current study, 15.4 and 24.2\%, respectively, of enrolled patients who received nivolumab were LTSs [2]. There was no observed difference in the baseline characteristics of the overall population and LTSs in the CheckMate 141 study [2]. In the current study, significant differences between non-2-year survivors and 2-year survivors in median age $(P=0.0227)$ and ECOG PS $(P=0.0001)$ were observed. A previous meta-analysis that investigated randomized clinical trials involving patients with cancer of several types reported that older patients ( $\geq 65$ years) could benefit more from immunotherapy than younger patients ( $<65$ years) [14]. In the current analysis, a significant difference in age at baseline was observed between 2-year survivors and non-2-year survivors; however, the difference was only 2 years between the median age of 2 -year survivors (67 years; range: $33-79$ ) and that of non-2-year survivors (65 years; range: $20-84$ ). Thus, it is unclear if this small but significant difference in age could be clinically meaningful.

In the current study, a significant difference was also observed in the baseline ECOG PS between 2-year survivors
Fig. 3 Distribution of irAEs in relation to onset and treatment status of nivolumab irAE immune-related adverse event

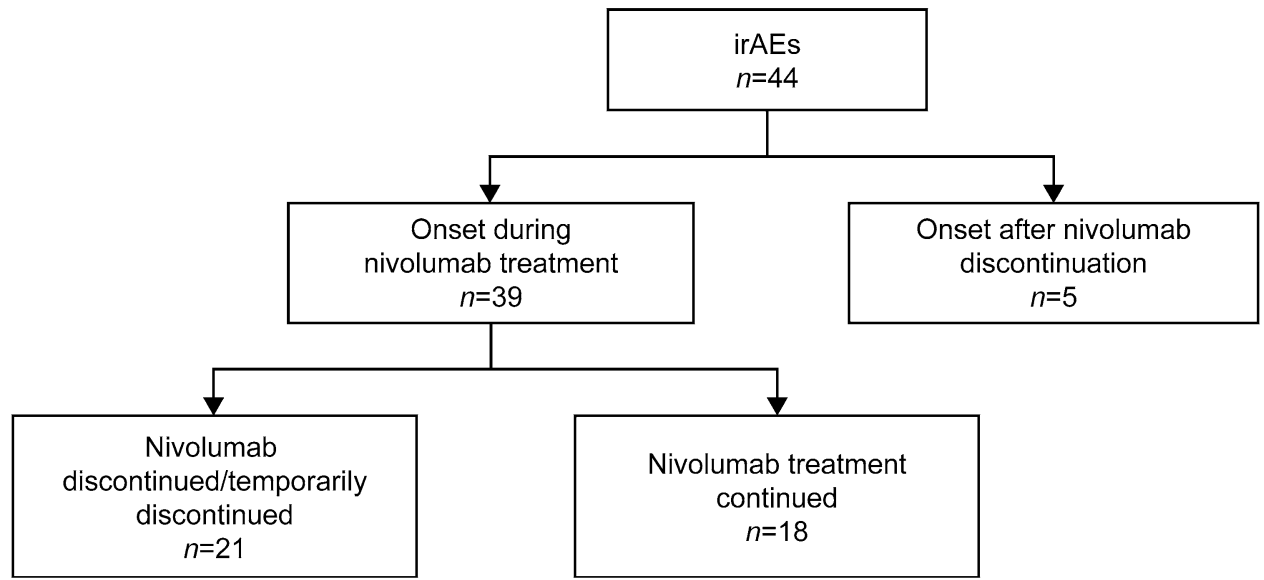


and non-2-year survivors. The previous 1-year follow-up analysis of this study showed that the ORR, PFS, and OS were numerically greater in patients with ECOG PS 0 than those in patients with ECOG PS 1 or $\geq 2$ [5]. Similarly, a difference by ECOG PS in the efficacy of immunotherapy has been reported in patients with malignant pleural mesothelioma [15], melanoma [16], and non-small-cell lung cancer [17] as well as for those with SCCHN [7]. Fushimi et al. investigated prognostic factors for patients with R/M SCCHN who received salvage chemotherapy after nivolumab treatment and reported that patients with ECOG PS 0 showed better OS compared with those with ECOG PS 1 [7]. Patients with a good ECOG PS can safely receive subsequent chemotherapy following immunotherapy compared with those with a poor ECOG PS. Moreover, since the 30-day mortality is known to increase in patients on palliative chemotherapy with a poor ECOG PS [18], the efficacy of subsequent chemotherapy could likely be influenced by ECOG PS. Further studies are warranted to confirm this hypothesis. Subgroup analyses by histological type confirmed that effectiveness was similar between patients with SCC and non-SCC, with no statistically significant differences. Age and ECOG PS remained significant prognostic factors in patients with SCC as well as in the overall population.

Several recent studies have demonstrated the efficacy of subsequent chemotherapy following immunotherapy in patients with $\mathrm{HNC}$ [6-9]. A previous study reported a significantly longer OS (7.8 months, $P=0.0028, n=25$ ) in Japanese R/M SCCHN patients who received salvage chemotherapy following nivolumab treatment than that in patients who received best supportive care (3.5 months, $n=31)$ [7]. In the current study, paclitaxel \pm cetuximab was the most frequently administered $(n=52)$ subsequent chemotherapy in patients who received chemotherapy following nivolumab. Median OS, median PFS, and ORR were 6.9 months, 3.5 months, and $35.7 \%$, respectively, in patients who received paclitaxel \pm cetuximab as subsequent chemotherapy. In a previous clinical study investigating the efficacy of a combination of paclitaxel and cetuximab as first-line treatment in patients with R/M SCCHN, median OS, median PFS, and ORR were 8.1 months, 4.2 months, and $54 \%$, respectively [19]. In general, a late-line palliative chemotherapy results in poor prognosis [20]. In the present study, despite $72.7 \%$ of patients receiving nivolumab as second- or later-line treatment [5], paclitaxel \pm cetuximab as subsequent chemotherapy demonstrated favorable effectiveness, which was consistent with the findings of a previous study that investigated a combination of paclitaxel and cetuximab as first-line treatment [19]. Several small retrospective studies in Japanese patients with R/M SCCHN have also reported the effectiveness of chemotherapy following treatment with immune checkpoint inhibitors [7-9].
Subsequent chemotherapy with or without cetuximab following treatment with immune checkpoint inhibitors, including nivolumab, demonstrated improved clinical responses, with overall response rates of $40.9 \%$ [8], 60.0\% [9], and $36 \%$ [7], median OS of 7.3 months [7] and 14.5 months [8], and median PFS ranging from 2.3 months to 5.4 months [7-9]. Although a small number of patients in the current study also received subsequent chemotherapy with S-1 or platinum-based therapy ( $n=13$ and 12, respectively), the median OS and PFS were comparable with that achieved with paclitaxel \pm cetuximab. Taken together with the findings from previous studies, these results support the higher chemosensitivity for subsequent therapies after immunotherapy. The higher effectiveness of chemotherapy following nivolumab treatment could be considered as one of the reasons for improved OS, as suggested by earlier studies [6, 21].

In the 1-year follow-up analysis, no new safety signals had been identified compared with CheckMate 141, with a median time to onset of irAEs of 8.7 weeks [5]. The irAE profile in the current study was largely consistent with that of the 1-year analysis. Kidney disorder was newly identified as an irAE and was reported in one patient. Late-onset irAEs are rare but have been reported following immunotherapy $[22,23]$. The delayed onset of irAEs could be associated with prolonged receptor occupancy irrespective of undetectable serum levels of the immune checkpoint inhibitor, which may cause persistent immune activation even after treatment cessation [24]. In this study, five patients reported irAEs after nivolumab discontinuation, reinforcing the importance of careful monitoring for irAEs in patients even after discontinuation of nivolumab treatment.

Retrospective observational studies have several limitations, including the absence of a control group. Furthermore, our data were based on the assessments by individual physicians during their routine clinical practice, which may not always be complete or provide information for comparison and may contain measurement errors. Moreover, we preferentially included study centers that had a high number of patients with R/M HNC to allow for recruitment of an optimal number of patients, which may have unintentionally introduced a selection bias in the study.

\section{Conclusions}

These 2-year follow-up results in Japanese patients with R/M HNC demonstrated that the real-world effectiveness and safety of nivolumab was consistent with that observed in CheckMate 141. Chemotherapy following nivolumab treatment may be effective in patients with R/M HNC.

Supplementary Information The online version contains supplementary material available at https://doi.org/10.1007/s10147-021-02047-y. 
Acknowledgements The authors thank Mebix (Tokyo, Japan) for operation of the study and statistical analysis in their role as the contract research organization, which was funded by Bristol-Myers Squibb K.K. and Ono Pharmaceutical Co., Ltd. The authors also thank Deepali Garg, MBBS, PGDHA, of Cactus Life Sciences (part of Cactus Communications, Mumbai, India) for providing medical writing support, which was funded by Bristol-Myers Squibb K.K. and Ono Pharmaceutical Co., Ltd.

Author's contributions AH, YS, and T. Yamada contributed to the study conception and design. RY, YS, NH, SK, T. Yokota, TF, KT, M. Ando, KH, TU, HH, ST, TO, DS, MY, AW, KO, T. Yamazaki, NM, NK, M. Arai, SY, TA, TN, and AH were involved in data acquisition. $\mathrm{NH}$ and $\mathrm{T}$. Yamada performed data analysis and interpretation. The first draft of the manuscript was written by $\mathrm{NH}$ and T. Yamada. All authors reviewed and commented on subsequent drafts of the manuscript. All authors read and approved the final manuscript. All named authors meet the International Committee of Medical Journal Editors (ICMJE) criteria for authorship for this article, take responsibility for the integrity of the work as a whole, and have given their approval for this version to be published.

Funding This work was funded by Bristol-Myers Squibb K.K. and Ono Pharmaceutical Co., Ltd.

\section{Declarations}

Conflict of interest R. Yasumatsu reports grants from Ono Pharmaceutical Co., Ltd. during the conduct of the study. N. Hanai, K. Omori, and A. Homma report grants from Bristol-Myers Squibb K.K. and Ono Pharmaceutical Co., Ltd. during the conduct of the study. T. Yokota reports an advisory role for Merck Biopharma and personal fees from Bristol-Myers Squibb K.K, Merck Biopharma, and Ono Pharmaceutical Co., Ltd. during the conduct of the study. K. Tsukahara reports personal fees from Bristol-Myers Squibb K.K. and Ono Pharmaceutical Co., Ltd. during the conduct of the study. S. Takahashi reports grants and personal fees from AstraZeneca K.K., Bayer, Bristol-Myers Squibb K.K., Chugai Pharmaceutical Co., Ltd., MSD, and Ono Pharmaceutical Co., Ltd. during the conduct of the study. T. Ueda reports a grant from Ono Pharmaceutical Co., Ltd. during the conduct of the study. T. Yamazaki reports grants from AstraZeneca K.K., Bristol-Myers Squibb K.K., and Ono Pharmaceutical Co., Ltd. during the conduct of the study. T. Yamada is an employee of Bristol-Myers Squibb K.K. T. Nekado is an employee of Ono Pharmaceutical Co., Ltd. Y. Shimizu, S. Kariya, T. Fujii, M. Ando, K. Hanyu, H. Hirakawa, T. Ono, D. Sano, M. Yamauchi, A. Watanabe, N. Monden, N. Kudo, M. Arai, S. Yonekura, T. Asakage, and M. Yoshida have nothing to disclose during the conduct of the study.

Open Access This article is licensed under a Creative Commons Attribution 4.0 International License, which permits use, sharing, adaptation, distribution and reproduction in any medium or format, as long as you give appropriate credit to the original author(s) and the source, provide a link to the Creative Commons licence, and indicate if changes were made. The images or other third party material in this article are included in the article's Creative Commons licence, unless indicated otherwise in a credit line to the material. If material is not included in the article's Creative Commons licence and your intended use is not permitted by statutory regulation or exceeds the permitted use, you will need to obtain permission directly from the copyright holder. To view a copy of this licence, visit http://creativecommons.org/licenses/by/4.0/.

\section{References}

1. Ferris RL, Blumenschein GJ, Fayette J et al (2016) Nivolumab for recurrent squamous-cell carcinoma of the head and neck. N Engl J Med 375:1856-1867

2. Ferris RL, Blumenschein GJ, Fayette $\mathbf{J}$ et al (2018) Nivolumab vs investigator's choice in recurrent or metastatic squamous cell carcinoma of the head and neck: 2-year long-term survival update of CheckMate 141 with analyses by tumor PD-L1 expression. Oral Oncol 81:45-51

3. Yen CJ, Kiyota N, Hanai N et al (2020) Two-year follow-up of a randomized phase III clinical trial of nivolumab vs. the investigator's choice of therapy in the Asian population for recurrent or metastatic squamous cell carcinoma of the head and neck (CheckMate 141). Head Neck 42:2852-2862

4. Pharmaceuticals and Medical Devices Agency Review Report (2017) Opdivo intravenous infusion. Available via https://www. pmda.go.jp/files/000223203.pdf. Accessed Aug 2021.

5. Hanai N, Shimizu Y, Kariya S et al (2021) Effectiveness and safety of nivolumab in patients with head and neck cancer in Japanese real-world clinical practice: a multicenter retrospective clinical study. Int J Clin Oncol 26:494-506

6. Saleh K, Daste A, Martin N et al (2019) Response to salvage chemotherapy after progression on immune checkpoint inhibitors in patients with recurrent and/or metastatic squamous cell carcinoma of the head and neck. Eur J Cancer 121:123-129

7. Fushimi C, Okamoto I, Matsuki T et al (2020) Salvage chemotherapy after nivolumab for recurrent or metastatic head and neck carcinoma. Anticancer Res 40:5277-5283

8. Kurosaki T, Mitani S, Tanaka K et al (2020) Safety and efficacy of cetuximab-containing chemotherapy after immune checkpoint inhibitors for patients with squamous cell carcinoma of the head and neck: a single-center retrospective study. Anticancer Drugs 32:95-101

9. Wakasaki T, Yasumatsu R, Uchi R et al (2020) Outcome of chemotherapy following nivolumab treatment for recurrent and/or metastatic head and neck squamous cell carcinoma. Auris Nasus Larynx 47:116-122

10. Grunwald V, Chirovsky D, Cheung WY et al (2020) Global treatment patterns and outcomes among patients with recurrent and/ or metastatic head and neck squamous cell carcinoma: results of the GLANCE H\&N study. Oral Oncol 102:104526

11. Postow MA, Sidlow R, Hellmann MD (2018) Immune-related adverse events associated with immune checkpoint blockade. $\mathrm{N}$ Engl J Med 378:158-168

12. Kariya S, Shimizu Y, Hanai N et al (2021) Effectiveness of nivolumab affected by prior cetuximab use and neck dissection in Japanese patients with recurrent or metastatic head and neck cancer: results from a retrospective observational study in a realworld setting. Int J Clin Oncol 26:1049-1056

13. Eisenhauer EA, Therasse P, Bogaerts J et al (2009) New response evaluation criteria in solid tumours: revised RECIST guideline (version 1.1). Eur J Cancer 45:228-247

14. Wu Q, Wang Q, Tang X et al (2019) Correlation between patient's age and cancer immunotherapy efficacy. Oncoimmunology. 8:e1568810

15. Fujimoto N, Okada M, Kijima T et al (2021) Clinical efficacy and safety of nivolumab in Japanese patients with malignant pleural mesothelioma: 3-year results of the merit study. JTO Clin Res Rep 2:100135

16. Nakamura Y, Kitano S, Takahashi A et al (2016) Nivolumab for advanced melanoma: pretreatment prognostic factors and early outcome markers during therapy. Oncotarget 7:77404-77415

17. von Pawel J, Bordoni R, Satouchi M et al (2019) Long-term survival in patients with advanced non-small-cell lung cancer treated 
with atezolizumab versus docetaxel: results from the randomised phase III OAK study. Eur J Cancer 107:124-132

18. Fiorin de Vasconcellos V, Rcc Bonadio R, Avanco G et al (2019) Inpatient palliative chemotherapy is associated with high mortality and aggressive end-of-life care in patients with advanced solid tumors and poor performance status. BMC Palliat Care 18:42

19. Hitt R, Irigoyen A, Cortes-Funes H et al (2012) Phase II study of the combination of cetuximab and weekly paclitaxel in the firstline treatment of patients with recurrent and/or metastatic squamous cell carcinoma of head and neck. Ann Oncol 23:1016-1022

20. Bourhis J (2005) New approaches to enhance chemotherapy in SCCHN. Ann Oncol 16(Suppl 6):vi20-vi24

21. Kacew AJ, Harris EJ, Lorch JH et al (2020) Chemotherapy after immune checkpoint blockade in patients with recurrent, metastatic squamous cell carcinoma of the head and neck. Oral Oncol 105:104676
22. Parakh S, Cebon J, Klein O (2018) Delayed autoimmune toxicity occurring several months after cessation of anti-PD-1 therapy. Oncologist 23:849-851

23. Weber JS, Hodi FS, Wolchok JD et al (2017) Safety profile of nivolumab monotherapy: a pooled analysis of patients with advanced melanoma. J Clin Oncol 35:785-792

24. Brahmer JR, Drake CG, Wollner I et al (2010) Phase I study of single-agent anti-programmed death-1 (MDX-1106) in refractory solid tumors: Safety, clinical activity, pharmacodynamics, and immunologic correlates. J Clin Oncol 28:3167-3175

Publisher's Note Springer Nature remains neutral with regard to jurisdictional claims in published maps and institutional affiliations.

\section{Authors and Affiliations}

\section{Ryuji Yasumatsu ${ }^{1} \cdot$ Yasushi Shimizu $^{2} \cdot$ Nobuhiro Hanai $^{3} \cdot$ Shin Kariya ${ }^{4} \cdot$ Tomoya Yokota $^{5} \cdot$ Takashi Fujii $^{6}$. Kiyoaki Tsukahara ${ }^{7}$. Mizuo Ando ${ }^{8,27}$. Kenji Hanyu ${ }^{9} \cdot$ Tsutomu Ueda $^{10}$. Hitoshi Hirakawa ${ }^{11}$. Shunji Takahashi ${ }^{12}$. Takeharu Ono ${ }^{13}$. Daisuke Sano ${ }^{14} \cdot$ Moriyasu Yamauchi ${ }^{15} \cdot$ Akihito Watanabe $^{16} \cdot$ Koichi Omori $^{17}$. Tomoko Yamazaki $^{18}$. Nobuya Monden ${ }^{19} \cdot$ Naomi Kudo $^{20} \cdot{\text { Makoto } \text { Arai }^{21} \cdot \text { Syuji Yonekura }}^{22} \cdot$ Takahiro Asakage $^{23}$. Takahiro Nekado $^{24}$ - Takayuki Yamada ${ }^{25}$. Akihiro Homma ${ }^{26}$ (1)}

1 Department of Otolaryngology, Graduate School of Medical Sciences, Kyushu University, Fukuoka, Japan

2 Department of Medical Oncology, Faculty of Medicine and Graduate School of Medicine, Hokkaido University, Sapporo, Japan

3 Department of Head and Neck Surgery, Aichi Cancer Center Hospital, Nagoya, Japan

4 Department of Otolaryngology, Head and Neck Surgery, Okayama University Hospital, Okayama, Japan

5 Division of Gastrointestinal Oncology, Shizuoka Cancer Center, Shizuoka, Japan

6 Department of Head and Neck Surgery, Osaka International Cancer Institute, Osaka, Japan

7 Department of Otorhinolaryngology, Head and Neck Surgery, Tokyo Medical University, Tokyo, Japan

8 Otorhinolaryngology and Head and Neck Surgery, The University of Tokyo Hospital, Tokyo, Japan

9 Head and Neck Oncology Center, International University of Health and Welfare, Mita Hospital, Tokyo, Japan

10 Department of Otorhinolaryngology, Head and Neck Surgery, Hiroshima University Hospital, Hiroshima, Japan

11 Department of Otorhinolaryngology, Head and Neck Surgery, University of the Ryukyus Hospital, Okinawa, Japan

12 Department of Medical Oncology, Cancer Institute Hospital of the Japanese Foundation for Cancer Research, Tokyo, Japan

13 Department of Otolaryngology, Head and Neck Surgery, Kurume University Hospital, Kurume, Japan

14 Otolaryngology, Head and Neck Surgery, Yokohama City University Hospital, Yokohama, Japan
15 Department of Otolaryngology, Head and Neck Surgery, Saga University Hospital, Saga, Japan

16 Department of Otolaryngology, Head and Neck Surgery, Keiyukai Sapporo Hospital, Sapporo, Japan

17 Department of Otolaryngology, Head and Neck Surgery, Kyoto University Hospital, Kyoto, Japan

18 Division of Head and Neck Cancer Oncology, Miyagi Cancer Center, Sendai, Japan

19 Department of Head and Neck Surgery, National Hospital Organization Shikoku Cancer Center, Matsuyama, Japan

20 Department of Otorhinolaryngology, Hirosaki University Graduate School of Medicine, Hirosaki, Japan

21 Department of Medical Oncology, Chiba University Hospital, Chiba, Japan

22 Department of Otorhinolaryngology, Head and Neck Surgery, Chiba University Hospital, Chiba, Japan

23 Department of Head and Neck Surgery, Tokyo Medical and Dental University Medical Hospital, Tokyo, Japan

24 Medical Affairs, Ono Pharmaceutical Co., Ltd, Osaka, Japan

25 Japan Medical and Development, Bristol-Myers Squibb K.K, Tokyo, Japan

26 Department of Otolaryngology, Head and Neck Surgery, Faculty of Medicine and Graduate School of Medicine, Hokkaido University, Kita15 Nishi7, Kita-Ku, Sapporo, Hokkaido 060-8638, Japan

27 Present Address: Department of Otolaryngology-Head and Neck Surgery, Okayama University Graduate School of Medicine, Okayama, Japan 